\title{
Collisionless damping of flows in the TJ-II stellarator
}

\author{
E Sánchez ${ }^{1}$, R Kleiber ${ }^{2}, \mathbf{R}$ Hatzky $^{3}$, M Borchardt ${ }^{2}$, \\ P Monreal ${ }^{1}$, F Castejón ${ }^{1}$, A López-Fraguas ${ }^{1}$, X Sáez $^{4}$, \\ J L Velasco ${ }^{1}$, I Calvo ${ }^{1}$, A Alonso ${ }^{1}$, D López-Bruna ${ }^{1}$ \\ 1 Laboratorio Nacional de Fusión, Asociación EURATOM-CIEMAT, Madrid, \\ Spain. \\ 2 Max-Planck-Institut für Plasmaphysik, EURATOM-Association, Greifswald, \\ Germany. \\ 3 Max-Planck-Institut für Plasmaphysik, EURATOM-Association, Garching, \\ Germany. \\ 4 Barcelona Supercomputing Center (BSC-CNS), Barcelona, Spain. \\ E-mail: edi.sanchez@ciemat.es
}

\begin{abstract}
The results of global linear gyrokinetic simulations of residual flows carried out with the code EUTERPE in the TJ-II three dimensional geometry are reported. The linear response of the plasma to potential perturbations homogeneous in a magnetic surface shows several oscillation frequencies: a GAMlike frequency, in qualitative agreement with the formula given by Sugama et al [1], and a much lower frequency oscillation in agreement with the predictions of Mishchenko et al $[2,3]$ for stellarators. The dependency of both oscillations with ion and electron temperatures and the magnetic configuration is studied. The low frequency oscillations are in the frequency range supporting the longrange correlations between potential signals experimentally observed in TJ-II.
\end{abstract}

PACS numbers: 52.30.Gz, 52.55.Hc, 52.65.Rr, 52.65.Tt, 52.25.Xz,

\section{Introduction}

The self-regulating capability of zonal flows (ZFs) in plasmas are now well recognized [4] and an effort to measure ZFs in experimental fusion devices has been done [5]. In some cases they have been identified with Geodesic-Acoustic-Mode type (GAM) oscillations $[1,6-9]$. Recently, long range correlations in potential have been measured in different fusion devices [10-14] that could be associated to ZFs.

The question of whether ZFs produced by turbulence can be sustained or are linearly damped was treated by Rosenbluth and Hinton [15]. They found that in a non collisional plasma the macroscopic flows are not completely damped, but a residual flow remains for long times. Afterwards, they extended the study to the collisional case [16] and treated in more detail the short time response. Other authors treated the problem in axisymmetric geometry $[1,17]$ and for stellarators [18-20]. In both Ref. [18] and Ref. [20] it was found that the optimization for neoclassical transport increases the residual zonal flow level in a stellarator. It was suggested [21,22] that a radial electric field can also affect the residual flow level. Gyro-kinetic simulations [23, 24] confirmed the influence of a radial electric field on the residual level. The collisionless 
response of a plasma to a potential perturbation in general stellarator geometry was treated in [2] where it was found that for long times the zonal flow does not reach a steady level but oscillates with a low frequency due to the radial drift of the locally trapped particles. This theory has afterwards been extended in [3] finding that the low frequency oscillations undergo a Landau damping.

In this work the results of global linear gyrokinetic simulations of collisionless development of flows in the three-dimensional geometry of the TJ-II stellarator are reported.

\section{Numerical method}

EUTERPE is a global gyrokinetic code aimed at simulating plasmas in three dimensional geometries [25-27]. It solves the gyroaveraged kinetic equation

$$
\frac{\partial f_{a}}{\partial t}+\dot{\mathbf{R}} \frac{\partial f_{a}}{\partial \mathbf{R}}+\dot{v}_{\|} \frac{\partial f_{a}}{\partial v_{\|}}=C\left(f_{a}\right)
$$

for the distribution function $f_{a}$ for up to three kinetic species, where $\dot{x}:=\frac{\mathrm{d} x}{\mathrm{~d} t}$.

In the electrostatic approximation, the equations of motion for the species $a(\dot{\mathbf{R}}$ and $\dot{v}_{\|}$) can be written as $\dot{\mathbf{R}}=\dot{\mathbf{R}}^{0}+\dot{\mathbf{R}}^{1}$ and $\dot{v}_{\|}=\dot{v}_{\|}^{0}+\dot{v}_{\|}^{1}$, with

$$
\begin{aligned}
\dot{\mathbf{R}}^{0} & =v_{\|} \mathbf{b}+\frac{\mu B+v_{\|}^{2}}{B_{a}^{*} \Omega_{a}} \vec{b} \times \nabla \mathbf{B}+\frac{v_{\|}^{2}}{B_{a}^{*} \Omega_{a}}(\nabla \times \mathbf{B})_{\perp} \\
\dot{\mathbf{R}}^{1} & =-\frac{\nabla\langle\phi\rangle \times \mathbf{b}}{B_{a}^{*}} \\
\dot{v}_{\|}{ }^{0} & =-\mu\left[\mathbf{b}+\frac{v_{\|}}{B_{a}^{*} \Omega_{a}}(\nabla \times \mathbf{B})_{\perp}\right] \nabla \mathbf{B} \\
\dot{v}_{\|}{ }^{1} & =-\frac{q_{a}}{m_{a}}\left[\mathbf{b}+\frac{v_{\|}}{B_{a}^{*} \Omega_{a}}\left(\mathbf{b} \times \nabla \mathbf{B}+(\nabla \times \mathbf{B})_{\perp}\right)\right] \nabla\langle\phi\rangle .
\end{aligned}
$$

The magnetic moment per unit mass, $\mu$, is a constant of motion $(\dot{\mu}=0) ; q_{a}$ and $m_{a}$ are the charge and mass respectively of the species $a, \Omega_{a}=q_{a} B / m_{a}$ is the cyclotron frequency, $\mathbf{b}=\mathbf{B} / B$ is the unit vector in the magnetic field $(\mathbf{B})$ direction and $B_{a}^{*}=B+\frac{m_{a} v_{\|}}{q_{a}} \mathbf{b} \cdot \nabla \times \mathbf{b} .\langle\phi\rangle$ is the gyro averaged potential introduced in Ref. [28].

In this work, collisions are not taken into account, so that $C(f)=0$ in Equation (1). The code uses a particle-in-cell scheme, the distribution function being discretized using markers whose trajectories are given by Equations (2). The $\delta f$ method is used, so that the distribution function is written as an equilibrium plus a timedependent perturbation as $f_{a}\left(\vec{R}, v_{\|}, \mu, t\right)=f_{a}^{0}\left(\vec{R}, v_{\|}, v_{\perp}\right)+\delta f_{a}\left(\vec{R}, v_{\|}, \mu, t\right)$. Using the $\delta f$ decomposition and linearizing the equation

$$
\frac{\partial \delta f_{a}}{\partial t}+\dot{\mathbf{R}}^{0} \frac{\partial \delta f_{a}}{\partial \mathbf{R}}+\dot{v}_{\|}{ }^{0} \frac{\partial \delta f_{a}}{\partial v_{\|}}=-\dot{\mathbf{R}}^{1} \frac{\partial f_{a}^{0}}{\partial \mathbf{R}}-\dot{v}_{\|}{ }^{1} \frac{\partial f_{a}^{0}}{\partial v_{\|}},
$$

is obtained. This equation is solved together with the quasineutrality equation that, assuming a long wavelength approximation $\left(k_{\perp} \rho_{i}<1\right)$ and adiabatic electrons, reads

$$
\left\langle n_{i}\right\rangle-n_{0}=\frac{e n_{0}(\phi-\bar{\phi})}{T_{e}}-\frac{m_{i}}{q_{i}} \nabla_{\perp}\left(\frac{n_{0}}{B^{2}} \nabla_{\perp} \phi\right),
$$

where $\bar{\phi}$ is the potential averaged over a flux surface. 

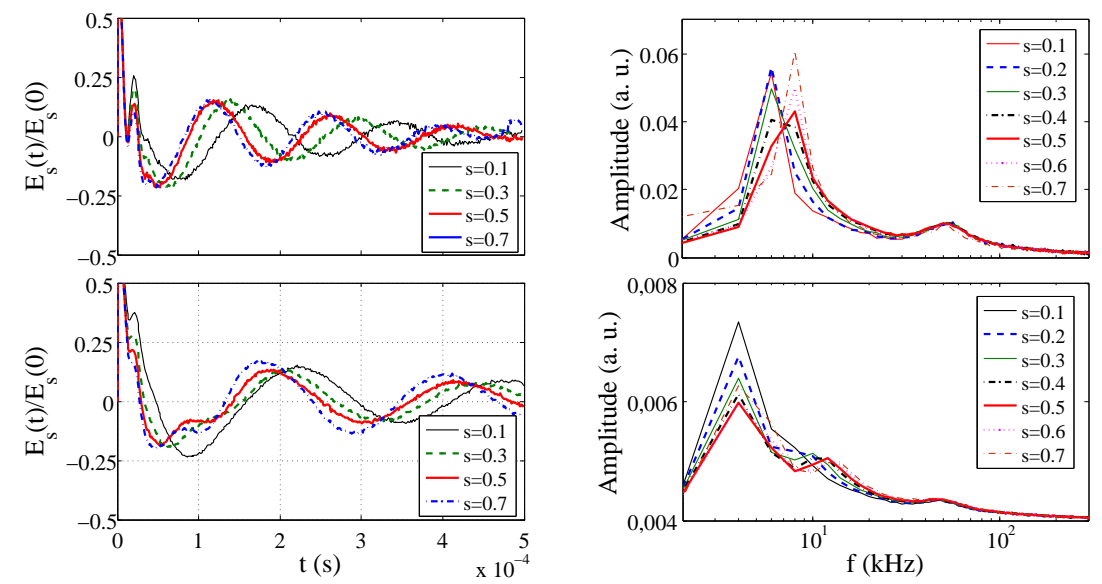

Figure 1: Left: Instantaneous radial electric field at four radial positions $(s)$ in the standard (top) and 32_102_65 configurations (bottom). Right: Spectra of the electric field oscillations in the left.

A local maxwellian distribution is used as equilibrium distribution function $f^{0}$. The simulations are initiated with a radius dependent perturbation of the form $\delta f \propto f_{M} \cos (\pi s)$, where $s$ is the normalized toroidal flux, used as a radial coordinate in the code and $f_{M}$ is a Maxwellian. The system is allowed to evolve and the radial electric field is monitored during the simulation at several radial positions.

\section{Simulation of residual flows in TJ-II}

TJ-II is a medium size stellarator $(R=1.5 \mathrm{~m}, 0.1<a<0.25,0.96<\iota<2.5)$ with low magnetic shear and a great flexibility in the magnetic configuration, that can be changed (even dynamically in the course of a plasma discharge) by changing the ratio of currents in the coils [29].

Gyro-kinetic simulations of residual flows have been carried out in two magnetic configurations of TJ-II: the standard configuration (100_44_64ł) and a highly indented (32_102_65) one. Both ion and electron temperatures profiles are flat with $T_{i}=T_{e}=$ $100 \mathrm{eV}$. Figure 1 shows the instantaneous electric field (normalized to its initial value) at four radial positions $(s)$ for both configurations and also the spectra of these oscillating traces, showing several oscillation frequencies.

In the standard configuration (Figure 1-top) a frequency is found around $50 \mathrm{kHz}$, in agreement with the formula given by Sugama et al [1] and Gao et al Gao08 for GAMs. A Low Frequency Oscillation (LFO) around 6-8 kHz, in qualitative agreement with the theory by Mishchenko et al [2], dominates the oscillations. The GAM oscillation frequency does not depend much on the radial position, while the LFO frequency is higher at the outer radial positions. The GAM oscillation is only visible in the first peak in Figure 1-left and disappears afterwards, which suggests a strong damping of the GAM oscillations as expected from the theory of GAMs for small values of the safety factor. In the standard configuration $t=1 / q$ is in the range

$\ddagger$ In TJ-II, the magnetic configurations are named according to the currents (in hundreds of A) in the circular, helical and vertical field coils respectively, following a pattern like ccc_hh_vv. 

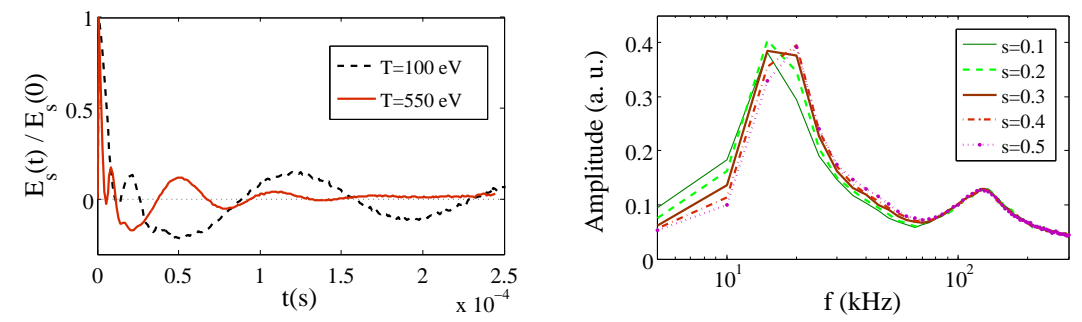

Figure 2: Oscillations in the radial electric field for $T=100$ and $550 \mathrm{eV}$ at $s=0.5$ (left) and spectra of the oscillations for the $T=550 \mathrm{eV}$ (right) in the standard configuration.

$1.55-1.65$. The LFO is slowly damped, in agreement with the theory of Helander et al [3], that predicts a slow Landau damping of the LFOs.

The residual zonal flow level at long times, as can be seen in Figure 1-(topleft $)$, is quite small as compared to that for a tokamak equivalent in size $\left(E_{\infty} / E_{0}=\right.$ $\left.\left(1+1.6 q^{2} \sqrt{R / r}\right)^{-1}\right)[15]$ that would give $E_{\infty} / E_{0} \approx 0.28$ at $s=0.5$. Whether this residual level is very small or exactly zero can not be concluded from these simulations because numerical instabilities appear for long times, probably due to the large number of particles that escape from the plasma.

The results for the configuration 32_102_65 are shown in Figure 1-(bottom). This configuration has a smaller volume, a larger rotational transform ( $t$ around 2.25), a lower trapped particle fraction and a deeper magnetic well, while has almost the same magnetic shear as the standard one. Comparing the results of both configurations (Figure 1) it is observed that the GAM oscillation appears less marked in this case. This is in agreement with the theory of GAMs, that predicts a damping of these oscillations decreasing with the safety factor $\left(\gamma_{G A M} \propto-e^{-c q^{2}}\right)[1,8]$. However, the frequency ot the GAM oscillation in the 32_102_65 configuration is smaller even though its rotational transform is higher. Probably, the expressions given by [1] and [8] for the GAM frequency derived for axisymmetric systems are not accurate enough for TJ-II with such high $t$ values. Expressions derived for the GAM frequency in earlier works with fluid [9] or kinetic models [6,7] are even less accurate for TJ-II.

The frequency of the LFO is slightly smaller in the highly indented configuration which is consistent with the fact that in this configuration, the plasma volume is more separated from the toroidal field coils and has a smaller trapped particle fraction.

Similar to the case of the standard configuration, the frequency of the GAM (around $46 \mathrm{kHz}$ ) does not depend much on the radial position. The LFO shows a peak around $4-5 \mathrm{kHz}$. .

\subsection{Dependency with temperature.}

3.1.1. Flat profiles. We have carried out linear simulations in the standard configuration, similar to the ones previously described but now with a higher temperature. The temperature and density profiles are flat with $T_{i}=T_{e}=550 \mathrm{eV}$ in this case. The instantaneous radial electric field at $s=0.5$ for both temperatures together with the spectra of its oscillations for several radial positions in the high temperature case are shown in Figure 2.

Both the GAM $(135-140 \mathrm{kHz})$ and the LFO $(15-20 \mathrm{kHz})$ frequencies increase 

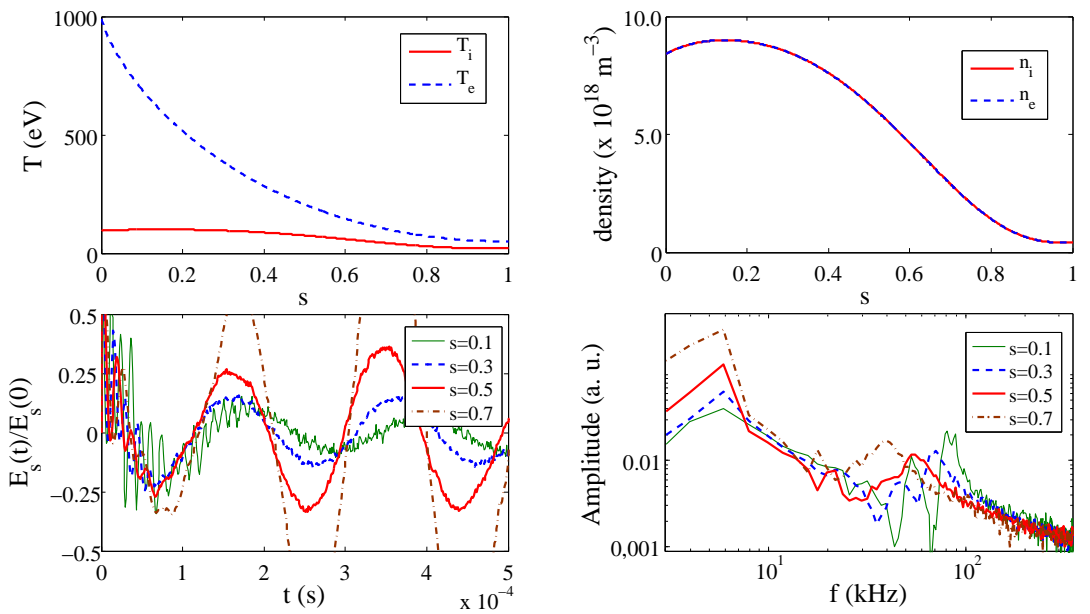

Figure 3: Top: Density (left) and temperature (right) profiles typical from ECRH plasmas in TJ-II. Bottom: Oscillations in the radial electric field versus time (left). Oscillations spectra (right).

with temperature, as can be appreciated by comparing the Figure 2 with Figure 1top-right. Both frequencies agree with a dependency with temperature as $f \propto T^{\frac{1}{2}}$. The damping of the LFO is larger in the high temperature case.

3.1.2. Experimental profiles. We have also carried out simulations using density and temperature profiles similar to those typical of ECRH heated discharges and used in neoclassical calculations [30]. The profiles are shown in the Figure 3-top. The results obtained in these simulations are shown in Figure 3-bottom.

The LFO is around $6 \mathrm{kHz}$ for all the radial positions. Recalling the theory of Mishchenko [2], both ions and electrons contribute to the oscillations in the electric field by their radial drift. However, in our simulations the electrons are considered adiabatic and do not contribute to this frequency; thus the LFO frequency can only depend on the ion orbits (temperature). The $T_{i}$ profile is rather flat as compared to the electron temperature profile. It has a slight decrease with radius that would have an effect in decreasing the LFO frequency, according to the dependency observed with flat profiles and described in the previous section. However, the trapped particle fraction, that increases radially, has an opposite effect on the zonal flow frequency. These two opposite effects seem to cancel each other, giving as a result a frequency of the zonal flow almost radius independent. The frequency of the GAM oscillations (40-100 kHz) depends on $T_{e}$ (through the radial dependency of $T_{e}$ ), in agreement with the GAM theory $[1,6-9]$.

\section{Conclusions and future work}

Linear gyrokinetic simulations of residual flows in the three dimensional geometry of TJ-II show a very small (compatible with zero) residual level in the zonal flow in different magnetic configurations. The relaxation of the radial electric field exhibits oscillations with several different frequencies. A high frequency oscillation appears, 
which is in agreement with the formula given by Sugama et al [1] for GAMs. This oscillation is quickly damped and its damping seems to increase with the rotational transform, in qualitative agreement with the theory [1,21]. A lower frequency oscillation, in the range $5-10 \mathrm{kHz}$ dominates over the GAM. This LFO zonal flow is also damped but on a much larger time scale and seems to be in qualitative agreement with the theory of Mishchenko et al [2].

For flat temperature profiles, the GAM frequency does not depend on the radial position while the zonal flow frequency increases with radius (trapped particle fraction), in agreement with [2]. The GAM frequency shows a dependency with electron temperature, in agreement with the theory of GAMs [1,6-9], while the LFO depends only on the ion temperature. This is in agreement with [2] if we take into account that in these simulations the electrons are considered to respond adiabatically, so that their radial drifts are not taken into account. Interestingly, the LFO are in the range of frequencies supporting the long range correlations experimentally observed between potential measurements of toroidally distant probes in the plasma edge of TJ-II prior to confinement transitions $[10,31]$, which suggests that these correlations could be related to the low frequency oscillations of zonal flows.

The validity of the collisionless approximation in TJ-II is limited to short times as compared to the typical collision time. The ion-ion collision time in ECRH heated discharges with the profiles shown in Figure 3 is in the range of $0.1-2 \mathrm{~ms}$, Taking this into account the comparison of the results of these collisionless simulations with experimental results can only be considered a qualitative indication.

Including kinetic electrons in the simulations would allow us to estimate the zonal flow frequency more accurately. Due to the high fraction of trapped particles in TJ-II, many particles escape from the plasma, preventing the continuation of the simulations. A better numerical treatment of these escaping particles would be necessary.

The radial structure of the zonal flow is very important for its interaction with the turbulence. The dependence of the plasma response on the radial wavelength of the initial perturbation will be considered in future works.

\section{Acknowledgments}

This work has been partially funded by the Spanish Ministry of Science and Innovation under projects ENE2008/06082/FTN and ENE2009-07247. The authors thankfully acknowledge the computer resources, technical expertise and assistance provided by the Barcelona Supercomputing Center and the Computing Center of CIEMAT.

\section{References}

[1] Sugama H. \& Watanabe T. 2006. Plasma Physics 72, 825.

[2] Mishchenko A. et al 2008 Phys. Plasmas 15072309.

[3] Helander P et al 2011 Plasma Phys. Control. Fusion 53054006.

[4] Diamond P. et al 2005 Plasma Phys. Control. Fusion 47 R161.

[5] Fujisawa A. 2009 Nuclear Fusion 4901300.

[6] Lebedev V. et al 1996. Phys. Plasmas 3, 3023.

[7] Novakovskii S. et al 1997. Phys. Plasmas 4, 4272.

[8] Gao Z. et al 2008 Phys. Plasmas 15072511.

[9] Winsor N. et al 1968 Physics of Fluids 112448.

[10] Pedrosa M A. et al 2008 Phys. Rev. Lett. 100215003.

[11] Hidalgo C. 2011 Plasma Phys. Control. Fusion 53074003.

[12] Xu Y. et al 2009 Phys. Plasmas 16110704. 
[13] Silva C. et al 2008 Phys. Plasmas 15120703.

[14] Manz P. et al 2009 Phys. Plasmas 16042309.

[15] Rosenbluth M N. and Hinton F L. 1998 Phys. Rev. Lett. 80724.

[16] Hinton F L and Rosenbluth M N. 1999 Plasma Phys. Control. Fusion 41 A653.

[17] Xiao Y and Catto P J. 2006 Phys. Plasmas 13082307.

[18] Sugama H and Watanabe T H. 2005 Phys. Rev. Lett. 94115001.

[19] Sugama H and Watanabe T H. 2006 Phys. Plasmas 13012501.

[20] Mynick H E and Boozer A H. 2007 Phys. Plasmas 14072507.

[21] Sugama H and Watanabe T-H. 2009 Phys. Plasmas 16056101.

[22] Watanabe T-H. et al 2008 Phys. Rev. Lett. 100195002.

[23] Kleiber R. et al 2010 Contributions to Plasma Physics 50766.

[24] Watanabe T H. et al 2011 Nuclear Fusion 51123003.

[25] Jost G. et al 2001 Phys. Plasmas 83333.

[26] Kornilov V. et al , 2004 Phys. Plasmas 113196.

[27] R. Kleiber and R. Hatzky. 2012 Computer Physics Communications 183305.

[28] Hahm T S. 1988 Phys. Fluids 312670.

[29] Alejaldre C. et al 1999 Plasma Phys. Control. Fusion 41 A539.

[30] Velasco J L. et al 2011 Plasma Phys. Control. Fusion 53115014.

[31] Alonso A. et al 2012 Submitted to Nuclear Fusion for publication. 